\title{
基于 MIMO 雷达的海面场景成像模拟及海浪 信息反演方法
}

\author{
王伟 ${ }^{(1)}$ ，张斌 (1)，马跃华 ${ }^{(2)}$, 朱昨庆 ${ }^{3}$ \\ (1) 哈尔滨工程大学自动化学院, 哈尔滨 150001 \\ (2) 上海机电工程研究所第十研究室, 上海 201109 \\ (3) 青岛产品质量监督检验研究院, 青岛 266001 \\ * 通信作者. E-mail: wangwei407@hrbeu.edu.cn
}

收稿日期: 2015-05-20; 接受日期: 2015-10-20; 网络出版日期: 2016-05-26

国家自然科学基金 (批准号: 61571148)、中国博士后特别资助 (批准号: 2015T80328)、中国博士后科学基金 (批准号: 2014M550182)、黑龙江省博士后特别资助 (批准号: LBH-TZ0410) 和哈尔滨市科技创新人才专项 (批准号: 2013RFXXJ016) 资助项目

摘要 多输入多输出 (multiple input multiple output, MIMO) 雷达作为一种新型的雷达体制, 其成像 兼具高分辨率与实时性的优点, 为海面成像观测提供了一种新的技术平台. 基于 MIMO 雷达的海面 成像技术目前还处于探索性研究阶段, 若千关键问题需要进一步解决和完善. 针对这一问题, 本文 从 MIMO 雷达海面场景模拟和基于 MIMO 雷达图像的海流与海浪信息反演两个方面展开了研究. 在 MIMO 雷达海面场景成像模拟方面, 针对目前利用 MIMO 雷达对海面进行成像的外场实验条件尚不 具备的问题, 提出一种基于双尺度模型的 MIMO 雷达海面场景成像模拟方法. 利用曲率修正的双尺 度方法和阴影效应构建海面的后向散射截面分布模型, 以此为目标采用舰载 MIMO 雷达成像系统与 反向投影成像算法对其作成像处理. 在海流与海浪信息反演方面, 利用所得的 MIMO 雷达图像反演 海表面流速与海浪参数。反演海流时, 采用加权最小二乘法, 在海浪参数反演时, 利用成像模拟仿真 实验确定了 MIMO 雷达的调制传递函数, 在此基础上获得了海浪谱与海浪参数反演结果. 相比于航 海导航雷达, 基于 MIMO 雷达的有效波高, 主波波向与上跨零周期反演都具有更高的精度. 仿真实验 验证了基于 MIMO 雷达的海面成像以及海流海浪信息反演相较于航海导航雷达的优越性.

关键词 MIMO 雷达 海面模拟 回波模拟 海流反演 海浪反演

\section{1 引言}

海面作为人们认识和研究海洋的基本媒介途径, 它反映了海洋的诸多信息, 如海浪、海面风场、浮 游生物以及海冰情况. 所以, 对海面进行实时观测成像并提取其中包含的海流海浪等信息对于国防、 航运、港口和海上石油平台建设以及海洋污染防治等具有重要的意义 ${ }^{[1,2]}$. 
利用雷达对海面成像属于海洋微波遥感的范畴. 雷达自带辐射源, 且所使用波段的电磁波几乎可 以无失真地穿透水汽云层, 所以与光学传感器相比, 雷达可以实现全天时、全天候观测, 且不受地理位 置限制 [3]. 目前, 用于海面成像的雷达主要分为合成孔径雷达和船用航海导航雷达两类.

合成孔径雷达 (synthetic aperture radar, SAR) 对海面成像覆盖范围广、分辨率高, SAR 遥感不仅 可以获得海浪信息, 而且可以间接获得海面风场、海底地形、海面浮油污染、海冰情况等 $[4,5] .1978$ 年 6 月美国宇航局 NASA 发射了 Seasat 卫星, 首次为人们提供了大量的微波海面遥感测量数据, 促进了 海洋遥感科学的发展 ${ }^{[6,7]}$. 经过多年的研究, 人们对 SAR 海浪成像的机理已经达成广泛的共识 ${ }^{[8 \sim 10]}$, 建立了海浪谱到 SAR 图像谱的非线性转换关系, 在此基础上研究了海浪信息反演技术. 合成孔径过 程是 SAR 获得高分辨率的途径, 同时却也是其受制约的根源所在. 合成孔径过程中海面的运动严重 影响了成像结果, 尤其是在高海况时. SAR 对海面成像实质是在 SAR 系统的相干时间和运动海面的 相干时间这两个时间内接收信号的积分过程, 因此 SAR 的分辨率受这两个相干时间的限制, 影响成像 的精度.

对海面成像和信息反演的另一途径是航海导航雷达等机械扫描式雷达. 20 世纪 50 年代, 人们发 现在海岸担任探测和警戒任务的雷达总是受到来自海面不明原因的 “干扰”. 1955 年, Crombie ${ }^{[11]}$ 观 测到这一现象, 并通过实验研究发现 “数十米波长的电磁波与海洋表面的相互作用, 将产生 Bragg 绕 射现象”. Wright ${ }^{[12]}$ 利用航海导航雷达作为波浪观测的工具, 由雷达影像直接进行波向、波长的判读. Enmenov 等研究了从雷达图像获取海浪波向、波长、波周期的方法 ${ }^{[13,14]} .20$ 世纪 80 年代 Hoogeboom 和 Ziemer 等将雷达图像数字化, 然后对图像数据进行二维 Fourier 变换, 并认为这就是海浪的二维波 数谱, 进一步求出海浪的频谱, 从而推算出海浪的参数 ${ }^{[15]}$. 利用航海导航雷达不仅可以反演海浪谱与

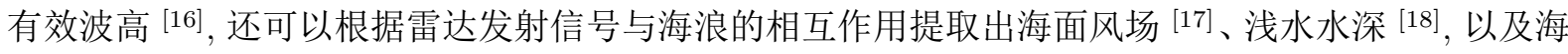
流信息 [19] 等. 然而, 由于航海导航雷达普遍采用的实孔径机械式扫描工作方式, 使得其在海面监测 中存在难以克服的缺陷. 航海导航雷达的方位角分辨率决定于实孔径天线的长度, 由于天线安装以及 载体条件的限制不可能采用很大孔径的天线, 所以导致航海导航雷达的方位向分辨率较差, 获得的雷 达图像精度不高. 另外, 航海导航雷达采用机械式扫描方式, 由于风速的影响会使得天线转速不均匀, 造成雷达图像中目标的位置与实际情况出现偏差, 影响了雷达图像与实际海面图像的对应程度; 机械 式扫描带来的另一个影响是对雷达图像采集时间的限制, 航海导航雷达的天线每转动一周形成一幅雷 达图像, 雷达图像采集时间受限于天线转速, 由于雷达天线的转速相对较慢, 在流速较大时很容易出现 角频率混叠的现象, 严重影响了海流反演精度, 甚至导致流速较大时普通的流速反演方法失效.

MIMO 雷达作为一种新型的雷达体制, 在成像方面同时具有高分辨率和实时性的优点, 所以利用 MIMO 雷达对海面进行实时监测具有很大的潜力. 由于 MIMO 雷达仍处于理论和样机研究阶段, 实 验用样机也多是通过分时复用的单发单收雷达合成的, 这种方式只能用于静止场景, 而对于时刻变化 的海面场景而言, 这种合成的多发多收方式不再适用. 另外, 海面变化是一个随机过程, 海态参数要受 到实际海况的限制, 具有不可控性, 这也增加了外场实验的困难. 在硬件条件受限和实测资料缺乏的情 况下, 通过成像模拟得到海面场景的 MIMO 雷达图像是一种重要的研究手段, 可以为海浪信息反演、 表面流反演、海面目标检测等研究提供基础.

本文将探讨海面场景 MIMO 雷达图像的模拟和提取 MIMO 雷达图像中包含的海面信息这两个 关键问题. 在 MIMO 雷达海面场景成像模拟方面, 提出一种基于双尺度模型的 MIMO 雷达海面场景 成像模拟方法. 利用曲率修正的双尺度方法和阴影效应构建海面的后向散射截面分布模型, 以此为目 标采用 MIMO 雷达成像系统与反向投影成像算法对其作成像处理, 而后对 MIMO 雷达海面回波数据 进行模拟. 在海流与海浪信息反演方面, 为了提取海面的海流与海浪信息, 利用所得的 MIMO 雷达图 


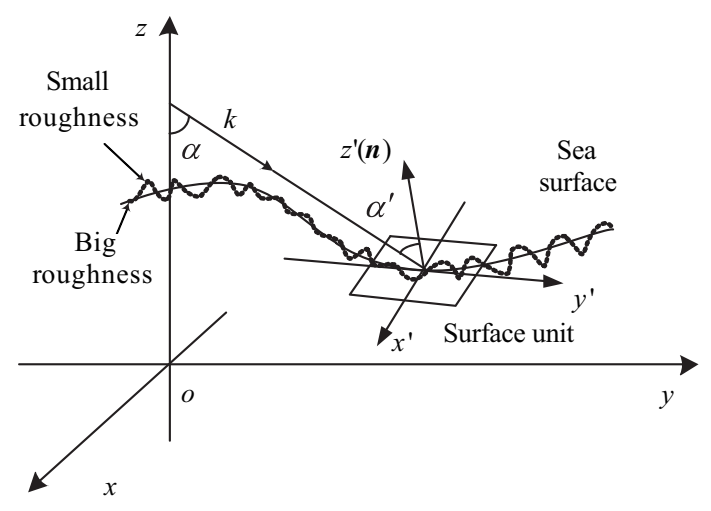

图 1 双尺度海面的电磁散射示意图

Figure 1 Electromagnetic scattering of two-scaled sea surface

像反演海表面流速与海浪参数. 反演海流时, 采用加权最小二乘法. 在海浪参数反演时, 利用成像模拟 仿真实验确定了 MIMO 雷达的调制传递函数, 在此基础上获得了海浪谱与海浪参数反演结果, 相比于 航海导航雷达, 基于 MIMO 雷达图像反演的有效波高、主波波向与上跨零周期都具有更高的精度. 仿 真实验验证了基于 MIMO 雷达的海面成像以及海流海浪信息反演相较于航海导航雷达的优越性.

\section{MIMO 雷达海面场景模拟}

\section{1 海面后向散射截面建模}

在海洋遥感中利用雷达对海面成像的实质就是利用回波信号估计出各个位置的分辨单元的后向 散射强度. 所以, 为了对海面进行成像, 首先需要确定海面各个面元的雷达后向散射截面大小. 本文讨 论单基地 MIMO 雷达在海面成像中的应用, 所以后向散射系数同雷达截面 (radar cross section, RCS) 是一个概念, 定义为 “特定方向每单位立体角上散射的功率和特定方向入射平面波在散射体单位面积 上功率之比乘以 $4 \pi \pi^{n}{ }^{[20]}$.

在三维海面模型的基础上, 利用曲率修正的双尺度方法和阴影调制函数, 可以计算各个面元后向 散射截面大小.

双尺度方法也称为组合表面法, 是将海面假设为由大尺度的重力波和小尺度的张力波组成, 小尺 度波依附在大尺度波上面. 海面每个微面元的散射是由小尺度波和电磁波的 Bragg 共振产生, 而大尺 度波通过倾斜调制改变电磁波的局部入射角, 从而改变后向散射截面的分布.

如图 1 所示, 在基准坐标系 $(x, y, z)$ 中, 海面高度分布可以表示为 $x$ 和 $y$ 的函数 $f(x, y)$. 假设电 磁波以平面波形式在 yoz 面内照射到粗粘海面上, 入射矢量为 $\boldsymbol{k}$, 全局入射角 $\alpha$ 定义为入射矢量与基 准坐标系 $z$ 轴的夹角. 海面微面元由于受到海面大尺度波的影响发生倾斜, 在微面元处建立本地坐标 系 $\left(x^{\prime}, y^{\prime}, z^{\prime}\right)$, 取本地坐标系的 $z^{\prime}$ 轴为微面元的法矢量, 设与入射矢量和微面元法矢量组成的平面垂 直的单位向量为本地坐标系的 $x^{\prime}$ 轴, 可以得到本地坐标矢量为

$$
\begin{gathered}
\boldsymbol{z}^{\prime}=\boldsymbol{n}=\frac{\left[-f_{x}-f_{y} 1\right]}{\left(1+f_{x}^{2}+f_{y}^{2}\right)^{1 / 2},} \\
\boldsymbol{x}^{\prime}=\frac{\boldsymbol{k} \times \boldsymbol{n}}{|\boldsymbol{k} \times \boldsymbol{n}|},
\end{gathered}
$$




$$
\boldsymbol{y}^{\prime}=x^{\prime} \times z^{\prime},
$$

其中, $f_{x}$ 和 $f_{y}$ 分别表示海面函数 $f(x, y)$ 在 $x$ 方向和 $y$ 方向的偏导, “ $\times$ ” 表示向量积运算符, $|\cdot|$ 表 示对向量取模.

设电磁波在 $y o z$ 平面内入射, $\boldsymbol{h}$ 和 $\boldsymbol{h}^{\prime}$ 分别为入射和反射电磁波的水平极化矢量, $\boldsymbol{v}$ 和 $\boldsymbol{v}^{\prime}$ 分别为 入射和反射电磁波的垂直极化矢量, 水平极化矢量 $\boldsymbol{h}, \boldsymbol{h}^{\prime}$ 和垂直极化矢量 $\boldsymbol{v}, \boldsymbol{v}^{\prime}$ 为

$$
\begin{gathered}
\boldsymbol{h}^{\prime}=\boldsymbol{x}^{\prime}, \boldsymbol{v}^{\prime}=-\left(\boldsymbol{y}^{\prime} \cos \varepsilon^{\prime}+\boldsymbol{z}^{\prime} \sin \alpha^{\prime}\right), \\
\boldsymbol{h}=\boldsymbol{x}, \boldsymbol{v}=-(\boldsymbol{y} \cos \alpha+\boldsymbol{z} \sin \alpha),
\end{gathered}
$$

其中, $\alpha^{\prime}=\cos ^{-1}\left(-\boldsymbol{k} \cdot \boldsymbol{z}^{\prime}\right)$ 为本地入射角, 即入射电磁波矢量与本地 $z^{\prime}$ 轴的夹角, “” 表示向量内积运 算符.

水平坐标为 $\left(x_{0}, y_{0}\right)$ 的倾斜面元在水平极化和垂直极化电磁波照射下的后向散射截面可以由下列 公式求得 ${ }^{[21]}$ :

$$
\begin{aligned}
& \sigma_{\mathrm{HH}}\left(x_{0}, y_{0}\right)=\left(\boldsymbol{h}^{\prime} \cdot \boldsymbol{h}\right) \sigma_{\mathrm{HH}}\left(\alpha^{\prime}\right), \\
& \sigma_{\mathrm{VV}}\left(x_{0}, y_{0}\right)=\left(\boldsymbol{v}^{\prime} \cdot \boldsymbol{v}\right) \sigma_{\mathrm{VV}}\left(\alpha^{\prime}\right),
\end{aligned}
$$

其中,

$$
\begin{gathered}
\sigma_{\mathrm{HH}}\left(\alpha^{\prime}\right)=8|\boldsymbol{k}|^{4} \delta^{2} \cos ^{4} \alpha^{\prime}\left|\frac{\varepsilon-1}{\left[\cos \alpha^{\prime}+\left(\varepsilon-\sin ^{2} \alpha^{\prime}\right)^{1 / 2}\right]^{2}}\right|^{2} S\left(2|\boldsymbol{k}| \sin \alpha^{\prime}, 0\right), \\
\sigma_{\mathrm{VV}}\left(\alpha^{\prime}\right)=8|\boldsymbol{k}|^{4} \delta^{2} \cos ^{4} \alpha^{\prime}\left|\frac{(\varepsilon-1)\left[\varepsilon\left(1+\sin ^{2} \alpha^{\prime}\right)-\sin ^{2} \alpha^{\prime}\right]}{\left[\varepsilon \cos \alpha^{\prime}+\left(\varepsilon-\sin ^{2} \alpha^{\prime}\right)^{1 / 2}\right]^{2}}\right|^{2} S\left(2|\boldsymbol{k}| \sin \alpha^{\prime}, 0\right),
\end{gathered}
$$

式中 $\boldsymbol{k}$ 为入射电磁波矢量, $\delta$ 为小尺度粗糙面的均方根高度, $\varepsilon$ 表示海水的复介电常数, $S\left(2|\boldsymbol{k}| \sin \alpha^{\prime}, 0\right)$ 是产生 Bragg 共振的海浪波数方向谱.

在传统的双尺度方法中忽略了入射波在表面上的绕射效应, 所以只适合用于表面大尺度成分变化 缓慢的情况. 当采用舰载或岸基雷达观测海面时, 属于低掠射角的情况, 不仅要考虑大尺度波的倾斜 调制作用, 还要考虑它的曲率调制作用 ${ }^{[22]}$.

采用岸基或舰载雷达进行海面观测时, 入射角较大, 某些面元会被其他的面元遮挡, 导致入射波 照射不到, 这就造成了阴影效应. 在进行大入射角或掠射角海面电磁散射仿真时必须要考虑这种影响. 在仿真时, 假设雷达高度和距离已知, 则对于特定的海面高程图, 可以根据几何关系判断面元是否被 遮挡.

对于高度为 $H$ 的雷达, 照射到海面上某一个微小面元 $q$ 处的入射角的计算公式为

$$
\alpha_{q}=\tan ^{-1} \frac{r_{q}}{H-\eta\left(x_{q}, y_{q}\right)},
$$

式中, $r_{q}$ 表示小面元 $q$ 到雷达天线的垂直距离. 在同一个方位向, 如果面元 $q$ 的与天线的水平距离 $r_{q}$ 比面元 $p$ 的水平距离 $r_{p}$ 大, 且入射角 $\alpha_{q}$ 不大于面元 $p$ 的入射角 $\alpha_{p}$ 时, 面元 $q$ 即被遮挡. 则经过阴 影调制之后, 海面各个面元后向散射系数为

$$
\sigma\left(x_{q}, y_{q}\right)= \begin{cases}0, & r_{q}>r_{p} \& \alpha_{q} \leqslant \alpha_{p} \\ \sigma\left(x_{q}, y_{q}\right), & \text { otherwise }\end{cases}
$$




\subsection{MIMO 雷达海面回波数据的模拟}

考虑单基地岸基或舰载 MIMO 雷达系统, 天线阵列分布方向定义为 $x$ 轴, 发射天线和接收天线 的 $x$ 坐标分别表示为 $u_{m}$ 和 $v_{n}$, xoy 面为海平面. MIMO 雷达采用多个天线同时发射相互正交的波 形, 第 $m$ 个发射信号为

$$
s_{m}(t)=p_{m}(t) \exp \left(\mathrm{j} 2 \pi f_{\mathrm{c}} t\right),
$$

式中, $p_{m}(t)$ 为发射信号的归一化包络, $f_{\mathrm{c}}$ 为载波频率.

假设被照射海面由 $Q$ 个微小面元组成, 每一个微小面元可以当作一个独立的散射点, 其后向散射 系数即由前面所提曲率修正双尺度方法求得, 其中任意一个面元 $q$ 处, 坐标为 $\left(x_{q}, y_{q}\right)$, 反射系数表示 为 $\beta_{q}$, 面元 $q$ 处的 MIMO 雷达照射信号为

$$
s_{q}(t)=\sum_{m=1}^{M} p_{m}\left(t-\tau_{m q}\right) \exp \left(2 \pi f_{\mathrm{c}}\left(t-\tau_{m q}\right)\right),
$$

式中, $\tau_{m q}$ 为第 $m$ 个发射阵元到 $q$ 点的传播时延, $\tau_{m q}=\sqrt{\left(x_{q}-u_{m}\right)^{2}+y_{q}^{2}} / \mathrm{c}$.

经过海面反射后, 第 $n$ 个接收阵元接收到的回波信号为所有面元反射信号的叠加, 表示如下:

$$
s_{n}(t)=\sum_{q=1}^{Q} \beta_{q} s_{q}\left(t-\tau_{q n}\right)=\sum_{q=1}^{Q} \sum_{m=1}^{M} \beta_{q} s_{m}\left(t-\tau_{m q n}\right),
$$

式中, $\tau_{q n}$ 为目标 $q$ 到第 $n$ 个接收阵元的传播时延, 发射接收双程时延为 $\tau_{m q n}$, 表示如下:

$$
\tau_{m q n}=\frac{\sqrt{\left(x_{q}-u_{m}\right)^{2}+y_{q}^{2}}+\sqrt{\left(x_{q}-v_{n}\right)^{2}+y_{q}^{2}}}{\mathrm{c}} .
$$

去除载波之后可以得到第 $n$ 个接收信号为

$$
s_{n}(t)=\sum_{q=1}^{Q} \sum_{m=1}^{M} \beta_{q} p_{m}\left(t-\tau_{m q n}\right) \exp \left(-\mathrm{j} 2 \pi f_{\mathrm{c}} \tau_{m q n}\right) .
$$

利用第 $m$ 个基频信号 $p_{m}(t)$ 对第 $n$ 个接收信号进行匹配滤波, 根据发射信号的正交特性, 匹配 滤波器的输出可以近似表示为

$$
s_{m n}(t)=\sum_{q=1}^{Q} \beta_{q} R_{m m}\left(t-\tau_{m q n}\right) \exp \left(-\mathrm{j} 2 \pi f_{\mathrm{c}} \tau_{m q n}\right),
$$

其中, $R_{m m}(\tau)$ 表示发射信号的自相关函数.

从上式可以看出, 当利用第 $m$ 个发射波形对第 $n$ 个接收信号进行匹配滤波可以分离出通道 $m \rightarrow n$ 的信号, 在接收端利用一组匹配滤波器对 $M$ 个发射信号进行分离, 可以得到经过 $M N$ 个不同通道的 信号, 相当于形成了一个阵元数为 $M N$ 的等效阵列, 大大扩展了阵列孔径, 使得 MIMO 雷达可以识别 更多的目标, 具有更高的角度分辨率等. 另外, 在 MIMO 雷达接收端进行匹配滤波的同时, 完成了距 离压缩. 在 MIMO 雷达等效阵列接收端进行方位向聚焦, 就可以完成距离角度的二维成像.

在远场假设条件下, 方位向聚焦可以通过波束形成的方法实现, 用公式表示如下:

$$
\boldsymbol{I}=\boldsymbol{S}^{\mathrm{T}} \boldsymbol{A},
$$


其中, $\boldsymbol{S}=\left[\boldsymbol{s}_{11}^{\mathrm{T}}(t) \boldsymbol{s}_{12}^{\mathrm{T}}(t) \cdots \boldsymbol{s}_{m n}^{\mathrm{T}}(t) \cdots \boldsymbol{s}_{M N}^{\mathrm{T}}(t)\right]^{\mathrm{T}}$ 为等效阵列接收信号矩阵, $\boldsymbol{A}$ 表示虚拟阵列流形 矩阵.

矩阵 $\boldsymbol{I}$ 即为海面在 MIMO 雷达上呈现的图像, 从 MIMO 雷达的成像过程可以看出, MIMO 雷达 全方向照射, 可以实现单次快拍成像, 没有孔径合成或波束扫描过程, 实时性好; 另外, MIMO 雷达通 过在接收端的波形分离, 形成一个远大于实际孔径大小的等效阵列, 与实孔径雷达相比, 提高了方位 向分辨率. MIMO 雷达的这些优点都使其非常适合用于对时刻运动的海面场景成像. 但由于 MIMO 雷 达是一种新型的雷达体制, 目前还不具备外场实验的条件, 所以本节利用电磁散射理论构建了海面的 后向散射截面分布图, 再利用 MIMO 雷达系统对其成像, 以模拟 MIMO 雷达海面场景成像的结果.

\section{3 基于 MIMO 雷达图像的海流海浪信息反演}

本节利用海面场景成像模拟得到的 MIMO 雷达图像反演海流和海浪信息. 首先对 MIMO 雷达图 像进行预处理, 包括内插变换与高通滤波, 然后介绍了基于加权最小二乘法的海流反演方法, 给出了 MIMO 雷达与航海导航雷达海流反演结果的对比. 最后, 建立了雷达图像谱与海浪谱之间的调制传递 函数, 得到海浪谱的反演结果, 进一步就可以利用反演得到的海浪谱求主要的海浪参数.

\subsection{MIMO 雷达图像预处理}

由于 MIMO 雷达与航海导航雷达采用的都是距离角度二维成像方式, 得到的雷达图像 $I(r, \theta, t)$ 都是以极坐标形式呈现的. 为了便于利用快速 Fourier 变换进行谱分析, 需要首先对雷达图像作内插 预处理, 形成 Decare 坐标系下的雷达图像 $I(x, y, t)$, 之后再采用三维 Fourier 变换获得雷达图像谱, 即 波数频率谱, 表示如下:

$$
F^{(3)}\left(k_{x}, k_{y}, \omega\right)=\int_{L x} \int_{L y} \int_{T} I(x, y, t) \exp \left(\mathrm{j}\left(k_{x} x+k_{y} y-\omega t\right)\right) \mathrm{d} x \mathrm{~d} y \mathrm{~d} t,
$$

式中 $L_{x}$ 和 $L_{y}$ 表示雷达图像在 $x$ 方向和 $y$ 方向的定义域, $T$ 表示雷达图像的采集时间长度.

在雷达图像谱中既包含了海浪信息, 也包含了许多不期望的频率分量. 其中主要的成分是由雷达 成像时的阴影调制和接收信号功率随距离的衰减引入的零频或者低频分量, 这些分量会给海流和海浪 信息反演带来误差, 需要先通过高通滤波加以抑制. 对雷达图像谱进行高通滤波可以用如下公式表示:

$$
F^{(3)}\left(k_{x}, k_{y}, \omega<3 \Delta \omega\right)=0,
$$

其中, $\Delta \omega$ 为角频率分辨单元, 由图像采集时间 $T$ 决定, $\Delta \omega=2 \pi / T$.

\section{2 基于 MIMO 雷达图像的海表面流反演}

海表面流估计是整个海面信息反演过程中的重要组成部分, 对后续的海浪参数反演具有重要的影 响. 本节介绍加权最小二乘海流反演方法, 然后给出在 $1 \mathrm{~m} / \mathrm{s}$ 和 $2 \mathrm{~m} / \mathrm{s}$ 下的二维海浪波数谱示意图. 最后将加权最小二乘海流反演方法应用于 MIMO 雷达图像序列, 获得了海表面流速的估值, 并与航海 导航雷达作对比.

在利用 MIMO 雷达对海面成像时, 可以认为雷达图像中包含的主要是海浪的回波信号, 所以雷达 的三维图像谱中的主要能量点, 即谱值较大的波数频率点处, 波数和频率应该满足色散关系. 在只考 
虑基本色散关系的情况下, 海表面流估计就是找出一条合适的色散曲线, 使得海浪的能量谱峰集中在 这条曲线附近, 进一步利用这条色散曲线求出流速矢量.

海浪的能量由色散方程联系起来. 深水条件时, 流速矢量为 $\left(u_{x}, u_{y}\right)$ 的线性波理论色散方程可以 表示为

$$
\omega=\sqrt{g k}+k_{x} u_{x}+k_{y} u_{y} .
$$

设三维图像谱中任意一个点, 三维坐标 $\left(k_{x 0}, k_{y 0}, \omega_{0}\right)$, 其图像谱幅值表示为 $F^{(3)}\left(k_{x 0}, k_{y 0}, \omega_{0}\right)$, 如果 图像谱幅值较大则可以认为该谱值是由海浪谱产生的, 其波数坐标 $\left(k_{x 0}, k_{y 0}\right)$ 与角频率坐标值 $\omega_{0}$ 之间 应该满足色散关系. 根据深水波条件下的基本色散关系, 可以利用波数 $\left(k_{x 0}, k_{y 0}\right)$ 与流速矢量 $\left(u_{x}, u_{y}\right)$ 求得一个估计的角频率, 如下式所示:

$$
\hat{\omega}_{0}=\sqrt{g k_{0}}+k_{x 0} u_{x}+k_{y 0} u_{y} .
$$

加权最小二乘法估计海流的基本思想就是在利用标准的最小二乘法求海表面流速时, 用三维图像 谱的谱能量对代价函数加权, 以提高海表面流速估计的准确性. 加权后的代价函数为

$$
J=\sum_{i=1}^{\mathrm{Num}}\left[\omega_{i}-\left(\sqrt{g k_{i}}+k_{x i} u_{x}+k_{y i} u_{y}\right)\right]^{2} W_{i},
$$

其中 Num 为选定的三维图像谱点个数, 图像谱点的选定标准通常为大于图像谱最大值的 $20 \%$. 可以 看出在选定的图像谱点范围内, 代价函数 $J$ 是关于流速 $u_{x}$ 和 $u_{y}$ 的函数, 令代价函数最小的 $u_{x}, u_{y}$ 组合即为最小二乘意义下的最优海流估值. 式 (23) 中权值 $W$ 定义如下:

$$
W_{i}= \begin{cases}F^{(3)}\left(k_{x i}, k_{y i}, \omega_{i}\right), & \omega_{i}>\omega_{t h}, \\ 0, & \text { otherwise }\end{cases}
$$

式中 $F^{(3)}\left(k_{x i}, k_{y i}, \omega_{i}\right)$ 即为三维图像谱值, 引入 $\omega_{t h}$ 是为了去除图像谱中的零频分量.

为了求出代价函数的极小点, 令其分别对 $u_{x}$ 和 $u_{y}$ 求偏微分并等于零. 可以推导出如下式子:

$$
\begin{aligned}
& \sum W_{i}\left(\omega_{i}-\sqrt{g k_{i}}\right) k_{x i}=\sum\left(W_{i} u_{x} k_{x i}^{2}+W_{i} u_{y} k_{x i} k_{y i}\right), \\
& \sum W_{i}\left(\omega_{i}-\sqrt{g k_{i}}\right) k_{y i}=\sum\left(W_{i} u_{y} k_{y i}^{2}+W_{i} u_{x} k_{x i} k_{y i}\right) .
\end{aligned}
$$

将式 (25) 与 (26) 结合在一起写为矩阵的形式, 整理可得加权最小二乘意义下的海表面流速估值, 如下式所示:

$$
\left[\begin{array}{l}
u_{x} \\
u_{y}
\end{array}\right]=\left[\begin{array}{cc}
\sum W_{i} k_{x i}^{2} & \sum W_{i} k_{x i} k_{y i} \\
\sum W_{i} k_{x i} k_{y i} & \sum W_{i} k_{y i}^{2}
\end{array}\right]^{-1} \sum\left[\begin{array}{l}
W_{i} k_{x i}\left(\omega_{i}-\sqrt{g k_{i}}\right) \\
W_{i} k_{y i}\left(\omega_{i}-\sqrt{g k_{i}}\right)
\end{array}\right] .
$$

上面式子中为了简便都省略了求和运算的上下限, 其下限默认为 $i=1$, 上限默认为 $i=$ Num.

图 2 给出了两种海流情况下沿正角频率积分形成的二维海浪波数谱, 图 $2(\mathrm{a})$ 中流速为 $1 \mathrm{~m} / \mathrm{s}$, 图 2(b) 中流速为 $2 \mathrm{~m} / \mathrm{s}$ 的情况. 这两种海浪波数谱都是在海流方向为 $45^{\circ}$, 海浪的主波方向为 $90^{\circ}$, 风 速为 $10 \mathrm{~m} / \mathrm{s}$ 的情况下获得的.

为了比较 MIMO 雷达和航海导航雷达海流反演结果, 本文利用成像模拟得到的雷达图像进行海 表面流速反演, 流速估计结果如表 1 所示. 其中利用最小二乘法反演海流时, 选取的谱值点的标准为 大于最大谱值 $20 \%$ 的点. 

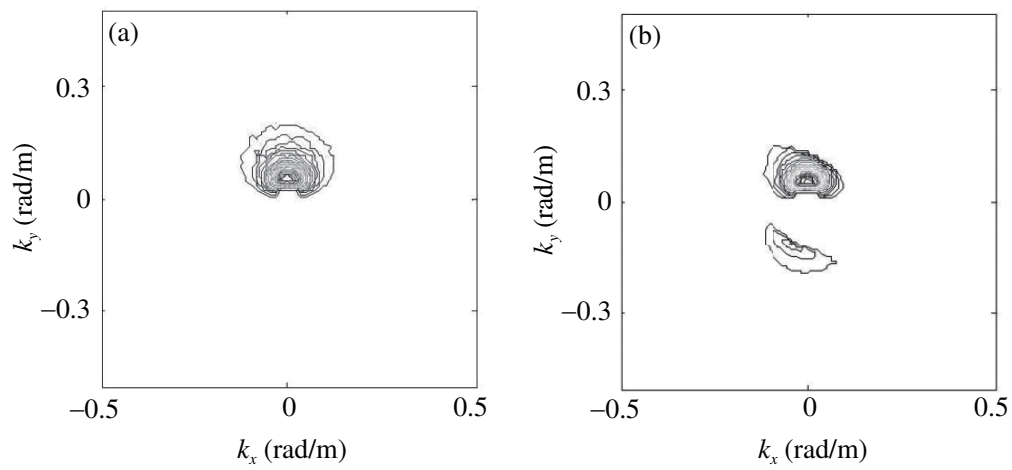

图 2 不同流速条件下二维海浪波数谱

Figure 2 2D wave number spectrum with different current speeds. (a) $u=1 \mathrm{~m} / \mathrm{s}$; (b) $u=2 \mathrm{~m} / \mathrm{s}$

表 1 流速为 $2 \mathrm{~m} / \mathrm{s}$, 流向为 $45^{\circ}$ 时的海流反演结果

Table 1 Current inversion result with current speed $2 \mathrm{~m} / \mathrm{s}$ and direction $45^{\circ}$

\begin{tabular}{ccc}
\hline & MIMO radar & Navigation radar \\
\hline$u_{x}$ estimation $(\mathrm{m} / \mathrm{s})$ & 1.1731 & 0.4798 \\
$u_{x}$ estimation $(\mathrm{m} / \mathrm{s})$ & 1.4535 & 1.0573 \\
Current speed estimation $(\mathrm{m} / \mathrm{s})$ & 1.8678 & 1.1611 \\
Current speed error $(\mathrm{m} / \mathrm{s})$ & -0.1322 & -0.8389 \\
Current direction estimation $\left(^{\circ}\right)$ & 51.0935 & 65.5916 \\
Current direction error $\left(^{\circ}\right)$ & 6.0935 & 20.5916 \\
\hline
\end{tabular}

从表 1 可以看出, 无论是流速估值或是流向估值, MIMO 雷达的估计误差都要比航海导航雷达小 很多, 这充分说明了 MIMO 雷达在海流反演中的优势, 而这是由于 MIMO 雷达更高的图像采样率与 更高的分辨率所决定的.

\section{3 基于 MIMO 雷达图像的海浪参数反演}

从海面场景的雷达成像过程可以看出, 由于倾斜调制和阴影调制的影响, 以及雷达图像插值变换 的影响, 得到的雷达图像并不是海面高程图, 所以雷达图像谱与实际的海浪谱之间存在着显著的差别. 为了由雷达图像谱得到海浪谱, 必须建立雷达图像谱与海浪谱之间的转换关系, 这个转换关系通常用 调制传递函数来描述.

调制传递函数 (modulating transfer function, MTF) 的概念最早由 Keller 等 ${ }^{[23]}$ 在 1975 年引入, 表示海面长波的轨道速度到雷达回波之间的线性频率响应函数, 描述了长波存在时雷达的后向散射对 海表面的线性响应. 具体定义如下式所示:

$$
|M(k)|^{2}=F(k) / S(k),
$$

其中 $F(k)$ 为雷达图像的一维波数谱, 可以通过雷达图像波数谱求得

$$
F(k)=\int_{-\pi}^{\pi} F^{(2)}(k, \theta) \mathrm{d} \theta=\int_{-\pi}^{\pi} k F^{(2)}\left(k_{x}, k_{y}\right) \mathrm{d} \theta .
$$




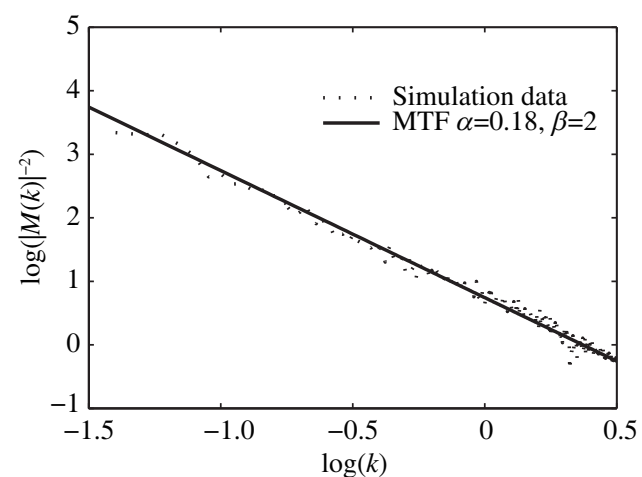

图 3 调制传递关系与 $\alpha k^{\beta}$ 曲线

Figure 3 Modulation transfer function and curve $\alpha k^{\beta}$

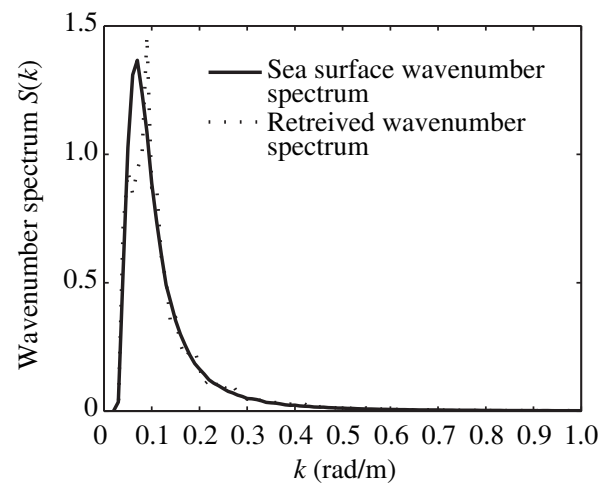

图 4 海面波数谱与反演波数谱

Figure 4 Sea surface wavenumber spectrum and the retrieved wavenumber spectrum

$S(k)$ 表示一维海浪波数谱, 在外场实验中通常由浮标测得的数据计算, 在本文中则可以由模拟海面时 采用的海浪谱直接求得.

大量的实验表明调制传递函数呈指数衰减, 调制传递函数的经验公式如下式所示:

$$
|M(k)|^{2} \propto k^{\beta}=\alpha k^{\beta} .
$$

为了获得准确的海浪谱与海浪参数反演结果, 需要对调制传递函数中的比例系数 $\alpha$ 和指数项 $\beta$ 进行 标定. 通常情况下, 调制传递函数被认为是只与雷达系统有关的, 所以可以通过实验对比实际的海面 海浪谱与雷达图像谱来标定调制传递函数的参数, 此后就可以使用标定好的调制传递函数进行海浪谱 反演.

由于 MIMO 雷达外场实验条件尚不具备, 在本文中采用成像模拟的方法获得海面场景的 MIMO 雷达图像, 这为利用仿真实验分析调制传递函数提供了条件. 本文通过比较成像模拟输入端的海面海 浪谱与输出端的雷达图像谱进行 MIMO 雷达调制传递函数标定.

图 3 给出了风速为 $10 \mathrm{~m} / \mathrm{s}$ 时的 MIMO 雷达图像谱与海浪谱之间的真实比例关系与经过标定的 调制传递函数曲线, 图中实线为由仿真数据计算得到的比例关系, 虚线为调制传递函数曲线, 参数为 $\alpha=0.18, \beta=2$. 图 4 给出了仿真海面时采用的海浪波数谱以及利用调制传递函数与雷达波数谱反演 得到的海浪波数谱.

从图 3 中可以看出由仿真数据计算得到的比例关系与调制传递函数曲线拟合良好, 符合指数项为 $\beta=2$ 的指数衰减形式. 其原因在于成像模拟中利用双尺度模型建立海面的后向散射截面模型, 由于 大尺度波对小尺度波的倾斜调制作用, 各个面元处的雷达图像强度正比于海面坡度, 这种海面成像方 式下的雷达图像谱反映的是海面的坡度谱而不是波高谱, 所以调制传递函数符合 $\beta=2$ 的形式. 从图 4 中可以看出模拟海面时使用的海浪波数谱与反演的波数谱比较吻合, 说明了所建立调制传递函数的 正确性, 为进一步反演海浪方向谱与海浪参数提供了条件.

利用带通滤波之后的二维图像谱以及标定好的调制传递函数, 可以获得海浪谱, 如下式所示:

$$
S\left(k_{x}, k_{y}\right)=F^{(2)}\left(k_{x}, k_{y}\right) /|M(k)|^{2}=\alpha^{-1} k^{-\beta} F^{(2)}\left(k_{x}, k_{y}\right) .
$$

利用不同海浪方向谱之间的转换关系, 可以得到不同形式的二维海浪谱为

$$
S(k, \theta)=k S\left(k_{x}, k_{y}\right)
$$



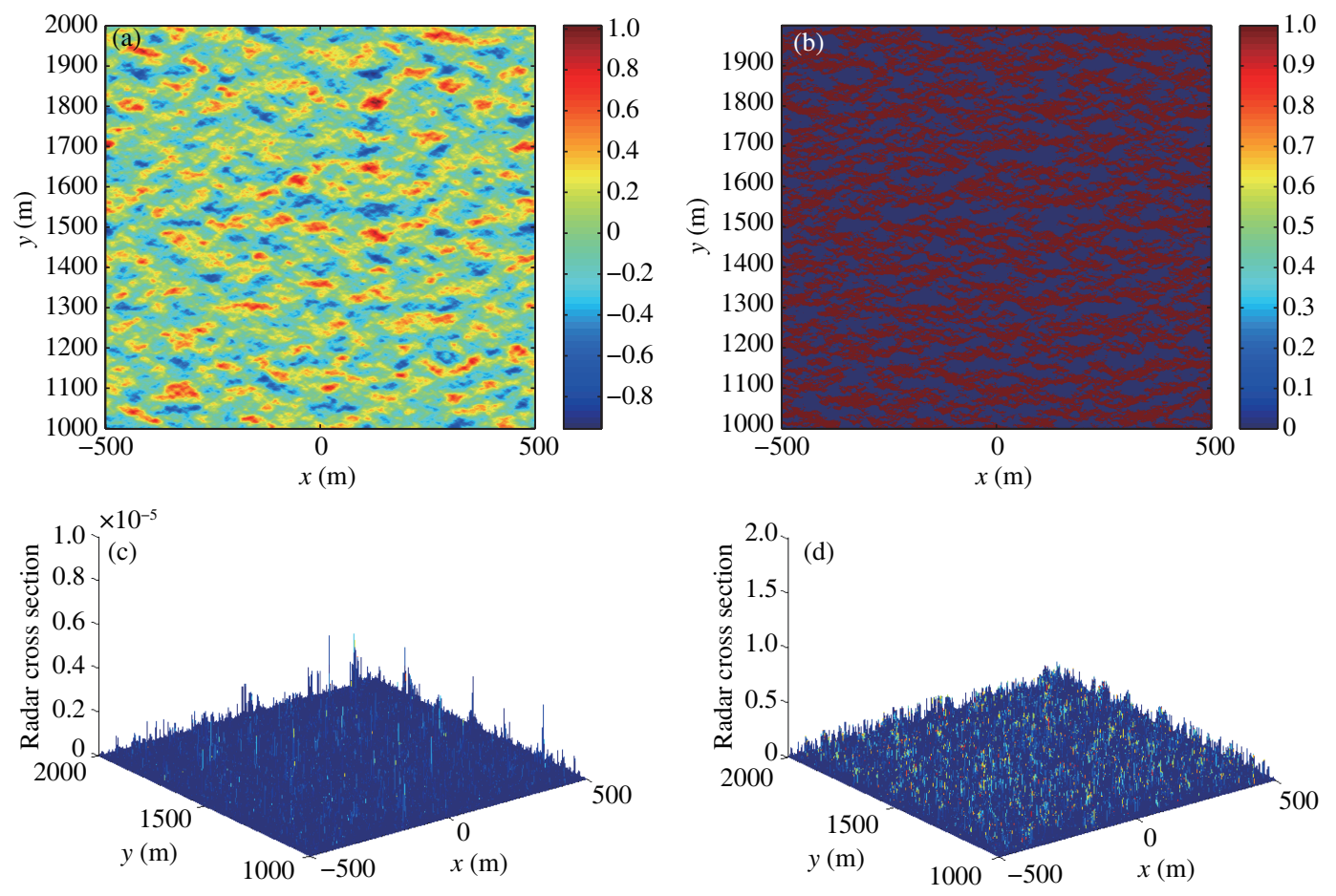

图 5 (网络版彩图) 风速为 $10 \mathrm{~m} / \mathrm{s}$ 时的海面电磁散射模型

Figure 5 (Color online) Electromagnetic scattering model of sea surface with wind speed $10 \mathrm{~m} / \mathrm{s}$. (a) Elevation drawing of simulated sea surface; (b) shadow function; (c) distribution diagram of RCS by HH polarization; (d) distribution diagram of RCS by VV polarization

$$
S(\omega, \theta)=\frac{S(k, \theta)}{\partial \omega / \partial k},
$$

式中 $\partial \omega / \partial k$ 表示对 $\omega$ 求 $k$ 的偏导, 忽略流速的影响, 可以写为

$$
\partial \omega / \partial k=\frac{1}{2} \sqrt{\frac{g}{k}} .
$$

对 $\theta$ 积分可以得到海浪能量相对于角频率的分布, 即一维角频率谱为

$$
S(\omega)=\int_{0}^{2 \pi} S(\omega, \theta) \mathrm{d} \theta .
$$

至此就得到了海浪谱的反演结果, 进一步可以利用反演得到的海浪谱求主要的海浪参数如有效波 高、平均周期、上跨零周期和平均波长等.

\section{4 仿真实验与分析}

\section{1 海面后向散射截面仿真}

对海面后向散射截面进行仿真. 假设雷达发射电磁波频率为 $10 \mathrm{GHz}$, 属于 X 波段, 天线高度为 $30 \mathrm{~m}$. 仿真时的海面区域选为 $x$ 方向 $-500 \sim 500 \mathrm{~m}, y$ 方向 $1000 \sim 2000 \mathrm{~m}$, 由 $1000 \times 1000$ 个微小 面元组成, 小面元尺寸为 $1 \mathrm{~m} \times 1 \mathrm{~m}$. 假设电磁波在 $y o z$ 平面内以平面波形式入射. 图 $5(\mathrm{a})$ 是风速为 

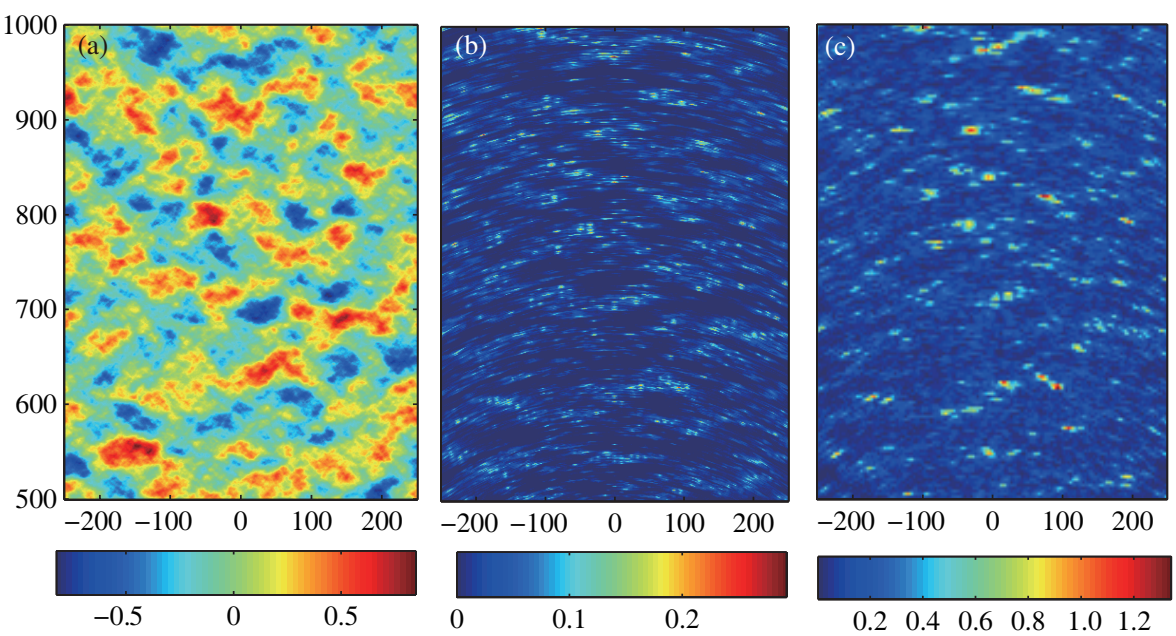

图 6 (网络版彩图) 模拟海面及其对应的雷达图像

Figure 6 (Color online) (a) Simulated sea surface; (b) MIMO radar image; (c) navigation radar image

$10 \mathrm{~m}$ 时由 Fung 海浪谱产生的海面, 图 5(b) 为根据海面与天线的几何关系计算得到的阴影调制函数 图像, 图 5(c) 为 $\mathrm{HH}$ 极化时由曲率修正双尺度方法与阴影效应共同作用产生的海面后向散射截面分 布图, 图 5(d) 为 VV 极化时得到的后向散射截面分布图.

比较图 5(c) 和 (d) 可以看出, 在相同的海况条件下, 垂直极化电磁波的海面后向散射系数要比水 平极化的后向散射系数大, 而水平极化后向散射系数分布图中则出现了很多类似尖刺的尖峰, 这就是 在雷达图像上出现的海尖峰现象, 可见海面后向散射截面的仿真结果与实际测量结果具有一致性, 验 证了所使用曲率修正双尺度方法建立海面电磁散射模型的正确性.

\section{2 海浪谱与海浪参数反演仿真实验}

本节通过仿真实验分析利用 MIMO 雷达图像反演海浪谱与海浪参数的有效性. 仿真采用的 MIMO 雷达系统与前述仿真时采用的 MIMO 雷达系统完全一致. 模拟海面时设海面 $10 \mathrm{~m}$ 高空平均风速为 $10 \mathrm{~m} / \mathrm{s}$, 主波波向为 $90^{\circ}$, 海表面存在一个流速大小为 $2.83 \mathrm{~m} / \mathrm{s}$, 流向为 $45^{\circ}$ 海流. 海面区域为 $x$ 方向 $-250 \sim 250 \mathrm{~m}, y$ 方向 $500 \sim 1000 \mathrm{~m}$, 海面面元大小为 $1 \mathrm{~m} \times 1 \mathrm{~m}$. 模拟的海面、MIMO 雷达图像以 及航海导航雷达图像如图 6 所示. 利用调制传递函数得到的海浪谱反演结果如图 7 所示, 其中 MIMO 雷达调制传递函数中 $\alpha=0.18$, 航海导航雷达调制传递函数中 $\alpha=6.5$. 图 8 则给出了实际海浪频谱 与反演得到的海浪频率.

比较图 8 中的海浪频谱, MIMO 雷达反演的一维海浪波数谱更接近于实际的一维海浪谱, 这是因 为 MIMO 雷达高分辨率与实时性造成的. 根据海浪参数与海浪谱之间的关系, 可以预期利用 MIMO 雷达图像反演海浪参数也会获得更准确的结果. 表 2 给出了由海浪谱计算得出的主要海浪参数, 可以 看出由 MIMO 雷达图像反演的有效波高、主波方向、上跨零周期误差更小, 体现了 MIMO 雷达在海 浪参数反演方面较航海导航雷达的优势.

\section{5 结论}

本文针对实际的 MIMO 雷达海面成像外场实验尚不具备条件的问题, 提出了一种 MIMO 雷达海 

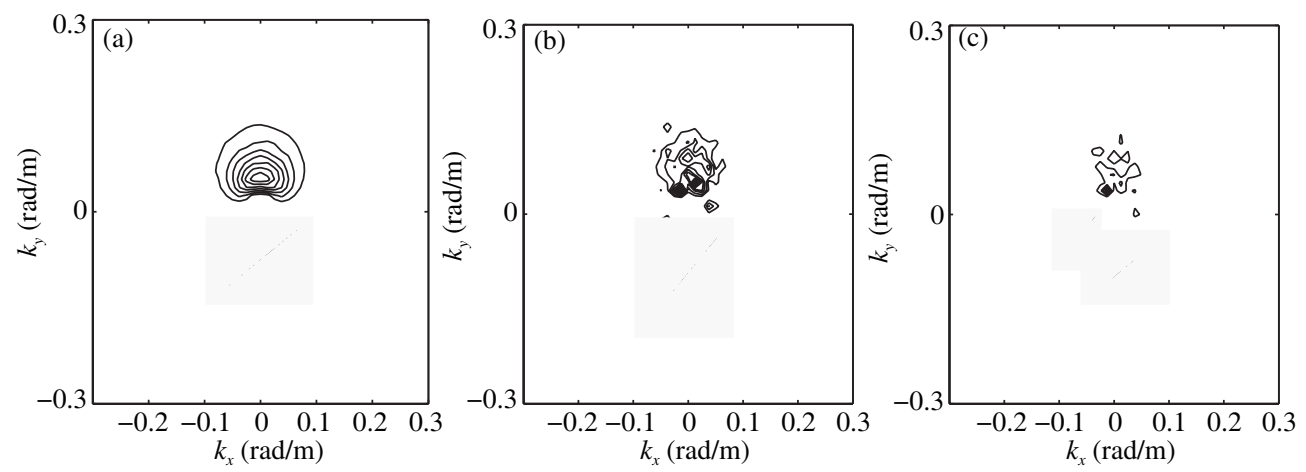

图 7 海面海浪方向谱与反演得到的海浪方向谱

Figure 7 Sea surface directional wave spectrum and retrieved directional wave spectrums. (a) Wave directional spectrum; (b) retrieved by MMO radar; (c) retrieved by navigation radar

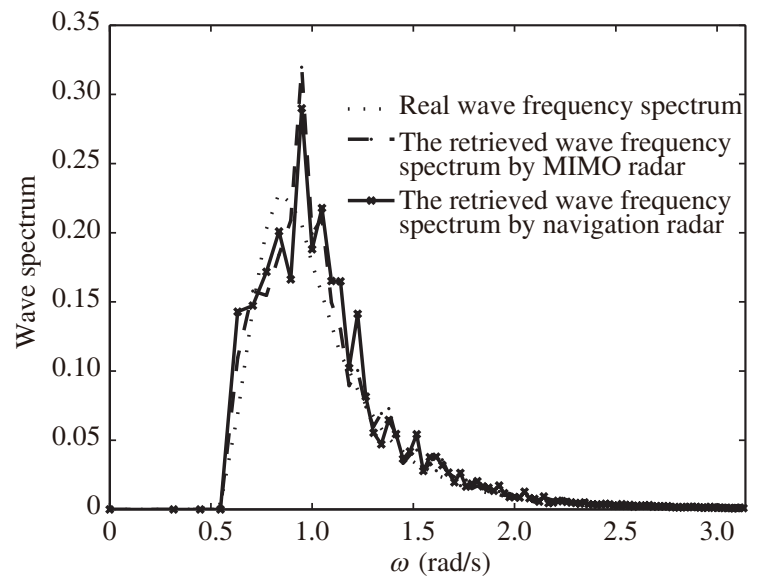

图 8 海浪频谱反演结果

Figure 8 The retrieved wave frequency spectrum

表 2 海浪参数反演结果

Table 2 Retrieved sea wave parameters

\begin{tabular}{cccccc}
\hline & Effective wave height $(\mathrm{m})$ & Frequency $(\mathrm{Hz})$ & Cycle $(\mathrm{s})$ & Direction $\left(^{\circ}\right)$ & Cross-zero cycle $(\mathrm{s})$ \\
\hline Real parameter & 1.46 & 0.13 & 7.5 & 90 & 5.88 \\
MIMO radar & 1.42 & 0.15 & 6.6 & 88.7 & 5.77 \\
Navigation radar & 1.54 & 0.15 & 6.6 & 86.5 & 5.72 \\
\hline
\end{tabular}

面场景成像模拟方法. 通过将海面电磁散射模型与 MIMO 雷达成像系统相结合, 模拟了 MIMO 雷达 的海面场景成像结果. 成像模拟结果显示了 MIMO 雷达图像具有与海面相似的纹理特征. 为了提取海 面图像中包含的海流与海浪信息, 利用 MIMO 雷达图像进行了海流与海浪信息的反演. 在海流反演 方面, 采用加权最小二乘法反演海流, MIMO 雷达的海流反演结果精度明显高于航海导航雷达. 在海 浪谱与海浪参数反演方面, 首先利用成像模拟的仿真实验确定了 MIMO 雷达的调制传递函数, 在此基 础得到的海浪参数反演结果表明, 相比于航海导航雷达, MIMO 雷达反演出的有效波高、主波波向与 上跨零周期都具有更高的精度. 海流与海浪反演结果显示了 MIMO 雷达在海面信息监测方面的优势. 


\title{
参考文献
}

1 Gerwick C, 金毅. 海洋工程设计手册: 海上施工分册. 上海: 上海交通大学出版社, 2013

2 俞聿修. 随机波浪及其工程应用. 第 3 版. 大连: 大连理工大学出版社, 2003

3 Kanevsky M B. Radar Imaging of The Ocean Waves. Boston: Elsevier, 2008

4 董庆, 郭华东. 合成孔径雷达海洋遥感. 北京: 科学出版社, 2005

5 杨永红. 合成孔径雷达海洋遥感导论. 北京: 海洋出版社, 2009

6 Apel J R. Seasat: a spacecraft views the marine environment with microwave sensors. In: Proceedings of OCEAN 75 Conference, San Diego, 1975. 803-815

7 Dunne J. The SEASAT-a project: an overview. In: Proceedings of OCEANS'76 Conference, Washington, 1976. $217-221$

8 Alpers W R, Ross D B, Rufenach C L. On the detectability of ocean surface waves by real and synthetic aperture radar. J Geophys Res: Oceans, 1981, 86: 6481-6498

9 Hasselmann K, Raney R K, Plant W J, et al. Theory of synthetic aperture radar ocean imaging: a MARSEN view. J Geophys Res: Oceans, 1985, 90: 4659-4686

10 何宜军. 成像雷达海浪成像机制. 中国科学 D 辑: 地球科学, 2000, 30: 554-560

11 Crombie D D. Doppler spectrum of sea echo at 13.56 Mc./s. Nature, 1955, 175: 681-682

12 Wright F F. Wave observation by shipboard radar. Ocean Sci Ocean Eng, 1965, 1: 506-514

13 Evmenov V F, Kozhukhov I V, Nichiporenko H T, et al. Test of the radar method of defining ocean waves elements. Fluid Mech Sov Res, 1973, 2: 141-145

14 Mattie M G, Harris D L. A System for Using Radar to Record Wave Direction. NASA Sti/recon Technical Report 79-1, 1979

15 Ziemer F, Rosenthal W, Carlson H. Measurements of directional wave spectra by ship radar. In: Proceedings of General Assembly Association Physics and Science of Oceans, Hamburg, 1983

16 Rosenthal W, Lehner S. Rogue waves: results of the MaxWave project. J Offshore Mech Arctic Eng, 2008, 130 : 021006

17 Dankert H, Horstmann J. A marine-radar wind sensor. In: Proceedings of IEEE International Symposium on Geoscience and Remote Sensing, Denver, 2006. 1296-1299

18 Flampouris S, Ziemer F, Seemann J. Accuracy of bathymetric assessment by locally analyzing radar ocean wave imagery. IEEE Trans Geosci Remote Sens, 2008, 46: 2906-2913

19 Senet C M, Seemann J, Flampouris S, et al. Determination of bathymetric and current maps by the method DiSC based on the analysis of nautical X-band radar image sequences of the sea surface (November 2007). IEEE Trans Geosci Remote Sens, 2008, 46: 2267-2279

20 Blunt S D, Chan T, Gerlach K. Robust DOA estimation: the reiterative superresoluton (RISR) algorithm. IEEE Trans Antenn Propag, 2011, 47: 332-346

21 郭立新, 王荵, 吴振森. 随机粗䊅面散射的基本理论与方法. 北京: 科学出版社, 2009

22 Voronovich A G, Zavorotny Y U. Curvature effects in the composite model for low-grazing-angle rough-surface scatter. Waves Random Media, 1998, 8: 41-52

23 Keller W C, Wright J W. Microwave scattering and the straining of wind-generated waves. Radio Sci, 1975, 10: $139-147$

\section{Sea surface imaging simulation and sea wave retrieval method based on MIMO radar}

\author{
Wei $\mathrm{WANG}^{1 *}$, Bin ZHANG ${ }^{1}$, Yuehua $\mathrm{MA}^{2}$ \& Zuoqing ZHU ${ }^{3}$ \\ 1 Automation Department, Harbin Engineering University, Harbin 150001, China; \\ 2 The 10th Laboratory, Shanghai Electro-mechanical Engineering Institute, Shanghai 201109, China; \\ 3 Qingdao Testing Institute of Product Quality Supervision, Qingdao 266001, China \\ *E-mail: wangwei407@hrbeu.edu.cn
}




\begin{abstract}
Multiple-input-multiple-output (MIMO) radar is an emerging radar system that has significant potential. MIMO radar can provide a higher resolution real-time imaging solution than traditional phased-array radar schemes and is well suited for the imaging of complex sea surfaces. MIMO radar imaging of sea scenes is still in the exploratory stage and several key issues need to be resolved and improved further. With the objective of achieving sea surface monitoring using MIMO radar, some basic research work on MIMO radar imaging simulation of sea surfaces, and MIMO radar sea current and wave information retrieval were conducted in this study. In order to analyze the feasibility and advantages inherent in MIMO radar imaging simulation of sea surfaces, ocean surface imaging based on MIMO radar was carried out via computer simulation. The curvature-modified two-scale method and shadowing modulation were used to build a back scatter section distribution model of a sea surface, which is taken as the object of MIMO radar imaging. In terms of sea current and wave information retrieval from MIMO radar images, the weighted least square method was used to fully explore the advantages of MIMO radar surface imaging. While retrieving the wave spectrum and wave parameters, the modulation transfer function of MIMO radar was determined via imaging simulation experiments. The retrieved significant wave height, main wave direction, and cross-zero cycle from MIMO radar images were found to be more precise than those from navigation radar images. The simulation results obtained also showed that MIMO radar sea surface imaging and parameter retrieval are superior to and more effective than marine radar.
\end{abstract}

Keywords MIMO radar, sea surface simulation, radar echo simulation, sea current retrieval, sea wave retrieval

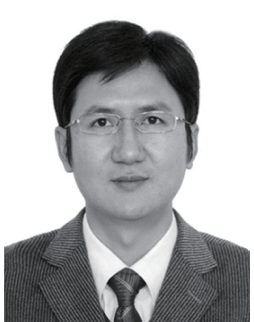

Wei WANG was born in 1979. He received a Ph.D. degree in navigation, guidance, and control from Harbin Engineering University. His research interests include radio navigation and radar signal processing. 